\title{
A comparative study on the performance of maximum likelihood, generalized least square, scale- free least square, partial least square and consistent partial least square estimators in structural equation modeling
}

\author{
Raudhah Zulkiflia, Nazim Aimran ${ }^{a^{*}}$, Sayang Mohd Deni ${ }^{\mathrm{a}}$ and Fatin Najihah Badarisam ${ }^{\mathrm{b}}$
}

${ }^{a}$ Center of Statistical and Decision Science Studies, Faculty of Computer and Mathematical Sciences, Universiti Teknologi MARA, 40450 Shah Alam, Selangor, Malaysia

${ }^{b}$ Faculty of Defense Science and Technology, National Defense University of Malaysia, 57000 Kuala Lumpur, Malaysia

C H R O N I C L E

Article history:

Received: October 20, 2021

Received in revised format: No-

vember 18, 2021

Accepted: December 27, 2021

Available online: January 2, 2022

Keywords:

Consistent partial least squares

Generalized least squares

Maximum likelihood

Scale-free least squares

Structural equation modeling

\section{A B S T R A C T}

Structural equation modeling offers various estimation methods for estimating parameters. The most used method in covariance-based structural equation modeling (CB-SEM) is the maximum likelihood (ML) estimator. The ML estimator is typically used when fitting models with normally distributed data. The growth of partial least squares path modeling (PLS-PM), including consistent partial least squares (PLSc), has also been noticed by researchers in the SEM fields. The PLSc has elevated interest in the scholastic setting in measuring the performance of various estimation methods in structural equation modeling. The choice of estimation methods has substantial impact in yielding parameter estimates. There could be a trade-off among the estimation methods' ability to deal with different types of data based on the model tested. Accordingly, this study aims to compare the performance of ML, generalized least squares (GLS), and scale-free least squares (SFLS) for CB-SEM as well as partial least squares (PLS) and consistent partial least squares (PLSc). Multivariate normal data were generated using Monte Carlo simulation with pre-determined population parameters and sample sizes using R Programming packages. To produce the estimated values, data analysis was performed using AMOS and SmartPLS for CB-SEM and PLS-SEM, respectively. The findings illustrate notable similarities between CB-SEM (ML) and PLS-SEM results when the true indicator loading is certainly high.

\section{Introduction}

The second-generation statistical analysis technique, structural equation modeling (SEM), is established for evaluating the inter-relationships among numerous variables in a model (Awang, 2015; Ainur et al., 2017). The covariance-based SEM (CBSEM) and variance-based SEM (VB-SEM) are the two most widely used methods in SEM (Henseler et al., 2016). PLS-SEM is presently the most fully developed of the VB-SEM approaches, commonly employed for fitting and testing hypotheses (McDonald, 1996; Schamberger et al., 2020). PLS-SEM has also been widely used in most social science fields (Hair et al., 2018). While CB-SEM is generally developed for confirmatory research, VB-SEM is known as a prediction-based approach to SEM that is mostly utilized for exploratory research (Sarstedt et al., 2014). The goal of CB-SEM is to estimate model parameters that minimize the discrepancies between the observed sample covariance matrix once the improved theoretical model has been validated (Awang, 2015). The normality of data distributions is necessary for several estimators in CB-SEM, which is rarely encountered in social sciences study. PLS-SEM, on the other hand, not only functions well with non-normal

* Corresponding author. Tel.: +60355435453

E-mail address: nazim@tmsk.uitm.edu.my (N. Aimran)

(C) 2022 by the authors; licensee Growing Science, Canada. doi: $10.5267 /$ j.ijdns.2021.12.015 
data but also has very few limitations when it comes to the application of ordinal and binary scales (Hair et al., 2017).

Apart from ML, several other estimators in CB-SEM have been developed to deal with different data characteristics, such as generalized least squares (GLS) and scale-free least squares SFLS (Hair. et al., 2017; Takane \& Hwang, 2018). Despite performing less well, the GLS fit function is able to minimize the disparities between a sample covariance matrix and the covariance matrix of a theoretical model like ML. Meanwhile, the SFLS fit function, which is derived from the GLS, is rarely used in past studies. Despite the widespread use of SEM in various fields, little has been researched on its estimation techniques (Andreassen et al., 2006). Thus, we think it is important to study the accuracy of estimation methods when fitting SEM models of different data characteristics. The development of PLSc by Dijkstra in 2010 was claimed to have resolved many SEM issues. The method is said to mimic the ML estimator in CB-SEM (Dijkstra \& Henseler, 2015) since it applies an attenuation adjustment to estimate factor loadings and path coefficients consistently (Schamberger et al., 2020). Therefore, we intend to examine whether PLSc performs better than the conventional PLS-PM and CB-SEM methods in measuring the parameter estimates. Towards this end, we used the comparative bias index (CBI) developed by Aimran et al. (2017b) and compared the indicator loadings estimations between ML, GLS, SFLS, PLS, and PLSc.

\section{Methodology}

\subsection{Research model for simulation}

In this study, we used the Monte Carlo Markov Chain (MCMC) simulation procedures to generate normally distributed data. We created three population models with different specifications of true indicator loadings. Each model had four latent constructs consisting of homogenous true indicator loading of $0.7,0.8$, and 0.9 and correlation of 0.7 , respectively. High indicator loading was set to avoid underestimation when using small sample sizes. Relationships between the constructs of the population were characterized as homogeneous. Sample sizes of 50, 100, 200, and 500 were opted, since PLS is commonly employed for small sample size (Dijkstra \& Henseler, 2015) and that a starting point of 100 to 200 is normally used as a sample size in path modeling (Awang, 2015). CB-SEM and PLS-SEM were employed to estimate the indicator loading using IBM AMOS version 24.0 and SmartPLS 3.0 respectively. Meanwhile, the R statistical programming environment was used to conduct the simulation procedure. The "psych" package, "MASS" package, "foreign" package, and the "semTools" package were used to generate multivariate normal data. Fig. 1, Fig. 2, and Fig. 3 present the population models that were tested with several estimation methods in CB-SEM and PLS-SEM.

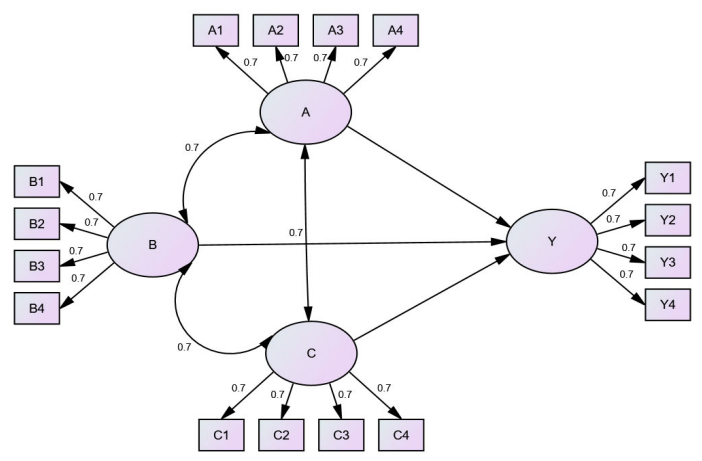

Fig. 1. Model 1

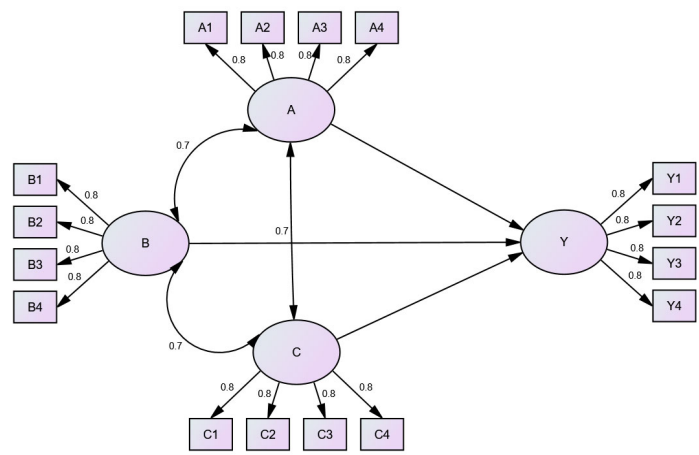

Fig. 2. Model 2

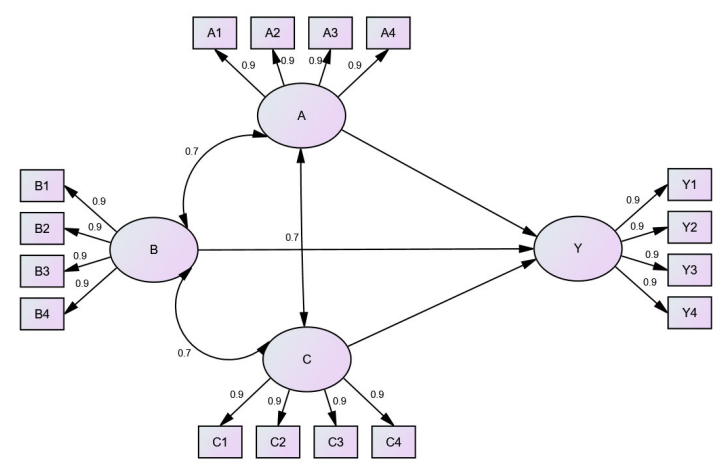

Fig. 3. Model 3 


\subsection{Estimation Methods}

\section{a. Maximum Likelihood (ML)}

For predicting fit and coefficients in CB-SEM, the Maximum Likelihood (ML) estimation approach is often used. ML utilizes derivatives to minimize the following fit function:

$$
F_{M L}=\log |\Sigma(\theta)|+\operatorname{tr}\left(S \Sigma^{-1}(\theta)\right)-\log |S|-(p+q)
$$

where the covariance matrix of the theoretical model denoted as $\Sigma$, and the sample covariance matrix defined as $S$. For a square matrix $\mathrm{B},|\mathrm{B}|$ implies the determinant of $\mathrm{B}$; $\operatorname{tr}(\mathrm{B})$ defines the sum of the diagonal elements of $\mathrm{B}$; and $(p+q)$ is the total numbers of manifest variables indicators. The derivation of fitting function of ML assumes that the observed variables are distributed normally (Newsom, 2018).

\section{b. Generalized Least Squares (GLS)}

According to Newsom (2018), generalized least squares is an additional fitting function. It reduces the disparity between sample covariance matrix, $S$ and covariance matrix, $\Sigma$. However, the GLS fit function uses a weight matrix for the residuals, defined as $W$. The fitting function is

$$
F_{G L S}=\left(\frac{1}{2}\right) \operatorname{tr}\left(\left\{\left[S-\Sigma(\theta) W^{-1}\right]\right\}^{2}\right)
$$

The simplicity of the function indicates that other weight matrixes could be applied to correct for the violations of distributional assumptions.

\section{c. Scale-free Least Squares (SFLS)}

The scale-free least squares estimation (SFLS) fitting function is

$$
F_{S F L S}=\left(\frac{1}{2}\right) \operatorname{tr}\left(\left\{\left[D-\Sigma(\theta) W^{-1}\right]\right\}^{2}\right)
$$

where $\mathrm{D}$ is the diagonal of sample covariance; $S$, the covariance matrix of the theoretical model, is denoted as $\Sigma$; and the weight matrix for the residuals is defined as $W$ (Arbuckle, 2011).

\section{d. Partial Least Squares (PLS)}

The PLS algorithm, according to Henseler et al. (2009), is a series of weight vectors regressions.

The iterative estimation of latent variable scores process is repeated until the maximum number of iterations has been reached. This step includes the following procedure:

By applying the factor weighting scheme inner weights were obtained:

$$
V_{j i}=\left\{\begin{array}{c}
\operatorname{cov}\left(Y_{j} ; Y_{i}\right) \text { if } Y_{j} \text { and } Y_{i} \text { are adjacent } \\
0 \text { otherwise }
\end{array}\right.
$$

where $Y_{j}$ is the latent variable (the dependent variable) and $Y_{i}$ is the latent variable (the independent variable) in the structural model.

Inside approximation:

$$
\tilde{Y}_{j}:=\sum_{i} b_{j i} Y_{i}
$$

where $\tilde{Y}_{j}$ is the computation for all constructs by utilizing the weighted sum of its adjacent constructs scores, $Y_{i}$; and $b_{j i}$ refers to the inner weights.

Outer weights; solve for Mode A block (reflective): 
$\tilde{Y}_{j n}=\sum_{k j} \widetilde{W}_{k j} X_{k j n}+d_{j n}$

Outer weights; solve for Mode B block (formative):

$$
X_{k j n}=\widetilde{W}_{k j} \tilde{Y}_{j n}+e_{k j n}
$$

Outside approximation:

$$
Y_{j n}:=\sum_{k j} \widetilde{W}_{k j} X_{k j n}
$$

where $X_{k j n}$ denotes the raw data for item $\mathrm{k}(\mathrm{k}=1, \ldots, \mathrm{K})$ of construct $\mathrm{j}(\mathrm{j}=1, \ldots, \mathrm{J})$; and observations $\mathrm{n}(\mathrm{n}=1, \ldots, \mathrm{N}), \tilde{Y}_{j n}$ is the construct scores from the inside; $\widetilde{W}_{k j}$ is the outer weights; $d_{j n}$ is the error term from a bivariate regression; and $e_{k j n}$ is the error term from a multiple regression. Under this , the updated weights (i.e., $\widetilde{W}_{k j}$ ) and the items (i.e., $X_{k j n}$ ) are linearly combined to renew the constructs scores (i.e., $Y_{j n}$ ).

\section{e. Consistent Partial Least Squares (PLSc)}

Dijkstra and Henseler (2015) developed consistent PLS (PLSc) to ensure that the PLS-SEM is adaptable while dealing with complicated models and distributional assumptions. In the correlation between two latent variables, the purpose is to correct for measurement error. To produce the deattenuated (i.e., consistent) correlation, PLSc refines the initial estimate.

$$
r_{i j}^{*}=\operatorname{cor}\left(\widetilde{\xi}_{l}, \widetilde{\xi}_{\jmath}\right)
$$

where $\xi$ is the latent variable scores. The new reliability coefficient must be utilized to assess the reliability of the construct scores, $\rho_{A}$, for each reflective construct, as presented in the following equation:

$$
\rho_{A}=\left(\widehat{w}^{\prime} \widehat{w}\right)^{2} \cdot \frac{\widehat{w}^{\prime}(S-\operatorname{diag}(S)) \widehat{w}}{\widehat{w}^{\prime}\left(\widehat{w} \widehat{w}^{\prime}-\operatorname{diag}\left(\widehat{w} \widehat{w}^{\prime}\right)\right) \widehat{w}}
$$

where $\widehat{w}$ denotes the outer weights estimates and $S$ is the sample covariance matrix. The correlation for attenuation is also needed if one of the constructs, $\widetilde{\xi}_{l}$ or $\widetilde{\xi}_{l}$, is formative. If both latent variables are formative, no modification is required.

$$
r_{i j}=\frac{r_{i j}^{*}}{\sqrt{\rho_{A}\left(\widetilde{\xi}_{l}\right) \cdot \rho_{A}\left(\widetilde{\xi}_{J}\right)}}
$$

For standardized coefficients based on correlations, the conventional OLS equation is expressed as follows:

$$
\beta=R_{X}^{-1} r_{X y}
$$

A vector of path coefficients denotes $\beta ; R_{X}$ is the correlation matrix of the indepedent variables of the structural equation; and the vector of correlations between the dependent variable and the independent variables denotes $r_{X y}$.

\subsection{Comparative Bias Index (CBI)}

As previously mentioned, the population data was generated based on prespecified parameters and different sample sizes. The population value was identified to be the actual model parameter (e.g., true indicator loading) values, which are required to create the simulation data. Hence, the CBI values for each item in the model were compared using the CBI described to evaluate the bias of simulation data parameter estimates as follows:

$$
C B I=1-\frac{|\hat{\theta}-\theta|}{\theta}
$$

where $\theta$ denotes the true value of the model parameter of interest and $\hat{\theta}$ is its estimate. A CBI value of $>0.9$ denotes unbiased or low bias of estimate, while a CBI value of $>0.8$ denotes acceptable bias of estimate. Otherwise, it is an unacceptable bias estimate.

\section{Result}

Table 1 to 3 summarize the performance of CB-SEM's and PLS-SEM's CBI values for all indicator loadings across the three prespecified models. 
Table 1

The Comparative Bias Index (CBI) - Model 1

\begin{tabular}{|c|c|c|c|c|c|c|}
\hline \multirow[t]{2}{*}{ Sample size } & \multirow[t]{2}{*}{ Items } & \multicolumn{3}{|c|}{$\begin{array}{c}\text { Comparative Bias Index } \\
\text { CB-SEM }\end{array}$} & \multicolumn{2}{|c|}{$\begin{array}{c}\text { Comparative Bias Index } \\
\text { PLS-SEM } \\
\end{array}$} \\
\hline & & ML & GLS & SFLS & PLS & PLSc \\
\hline \multirow[t]{16}{*}{50} & A1 & .886 & .971 & .814 & .963 & .637 \\
\hline & A2 & .986 & .729 & .957 & .836 & .903 \\
\hline & A3 & 1.000 & .986 & .843 & .927 & .681 \\
\hline & A4 & .914 & .943 & .757 & .824 & .626 \\
\hline & B1 & .771 & .943 & .757 & .857 & .600 \\
\hline & B2 & .914 & .429 & .986 & .977 & .923 \\
\hline & B3 & .814 & .843 & .800 & .990 & .744 \\
\hline & B4 & .929 & .857 & .900 & .983 & .939 \\
\hline & $\mathrm{C} 1$ & .943 & .057 & .986 & .931 & .959 \\
\hline & $\mathrm{C} 2$ & .757 & -.257 & .800 & .917 & .780 \\
\hline & $\mathrm{C} 3$ & .843 & -.729 & .714 & .893 & .553 \\
\hline & $\mathrm{C} 4$ & .886 & -.029 & .843 & .810 & .796 \\
\hline & Y1 & .543 & .371 & .586 & .841 & .534 \\
\hline & Y2 & .543 & .114 & .529 & .741 & .559 \\
\hline & Y3 & .829 & .571 & .871 & .907 & .851 \\
\hline & Y4 & .829 & .800 & .843 & .801 & .810 \\
\hline \multirow[t]{16}{*}{100} & A1 & .743 & .786 & .686 & .934 & .573 \\
\hline & A2 & .943 & .971 & .971 & .829 & .947 \\
\hline & A3 & .914 & .900 & .986 & .869 & .967 \\
\hline & A4 & .814 & .800 & .757 & .786 & .687 \\
\hline & B1 & .871 & .900 & .871 & .909 & .859 \\
\hline & B2 & .914 & .986 & .943 & .954 & .949 \\
\hline & B3 & .971 & .900 & 1.000 & .856 & .983 \\
\hline & B4 & .971 & .971 & .971 & .947 & .990 \\
\hline & $\mathrm{C} 1$ & .843 & .857 & .843 & .983 & .843 \\
\hline & $\mathrm{C} 2$ & .986 & .929 & .957 & .916 & .946 \\
\hline & $\mathrm{C} 3$ & .900 & .971 & .786 & .870 & .671 \\
\hline & $\mathrm{C} 4$ & .886 & .986 & .943 & .976 & .981 \\
\hline & Y1 & .843 & .900 & .843 & .984 & .837 \\
\hline & Y2 & .857 & .857 & .857 & .979 & .867 \\
\hline & Y3 & .986 & .986 & .986 & .859 & .977 \\
\hline & Y4 & .900 & .929 & .929 & .946 & .931 \\
\hline \multirow[t]{16}{*}{200} & A1 & .900 & .886 & .871 & .929 & .831 \\
\hline & A2 & .986 & .986 & .986 & .869 & .980 \\
\hline & A3 & .986 & 1.000 & 1.000 & .896 & .997 \\
\hline & A4 & .971 & .929 & .957 & .864 & .926 \\
\hline & B1 & .914 & .929 & .943 & .906 & .950 \\
\hline & B2 & .900 & .943 & .886 & .971 & .880 \\
\hline & B3 & .986 & .971 & .971 & .859 & .943 \\
\hline & B4 & .871 & .886 & .900 & .983 & .920 \\
\hline & $\mathrm{C} 1$ & .943 & .971 & .943 & .934 & .953 \\
\hline & $\mathrm{C} 2$ & .986 & .929 & .971 & .873 & .954 \\
\hline & $\mathrm{C} 3$ & .843 & .929 & .771 & .921 & .699 \\
\hline & $\mathrm{C} 4$ & .986 & .986 & .986 & .916 & .953 \\
\hline & Y1 & .971 & .986 & .971 & .920 & .994 \\
\hline & Y2 & .957 & 1.000 & .914 & .904 & .890 \\
\hline & Y3 & .943 & .929 & .914 & .847 & .897 \\
\hline & Y4 & .957 & .957 & .929 & .906 & .901 \\
\hline \multirow[t]{16}{*}{500} & A1 & .929 & .943 & .914 & .920 & .890 \\
\hline & A2 & 1.000 & 1.000 & .986 & .880 & .983 \\
\hline & A3 & .986 & .986 & .986 & .883 & .989 \\
\hline & A4 & .943 & .943 & .929 & .843 & .923 \\
\hline & B1 & .914 & .929 & .914 & .933 & .913 \\
\hline & B2 & .957 & .957 & .943 & .911 & .927 \\
\hline & B3 & 1.000 & 1.000 & 1.000 & .880 & .994 \\
\hline & B4 & .943 & .957 & .957 & .911 & .977 \\
\hline & $\mathrm{C} 1$ & .943 & .971 & .929 & .916 & .923 \\
\hline & $\mathrm{C} 2$ & .971 & .957 & .957 & .876 & .956 \\
\hline & $\mathrm{C} 3$ & .943 & .957 & .914 & .894 & .901 \\
\hline & $\mathrm{C} 4$ & .929 & .929 & .943 & .937 & .957 \\
\hline & Y1 & .971 & .971 & .971 & .903 & .979 \\
\hline & Y2 & .971 & 1.000 & .971 & .891 & .970 \\
\hline & Y3 & .986 & .986 & 1.000 & .889 & .980 \\
\hline & Y4 & 1.000 & .986 & .986 & .869 & .966 \\
\hline
\end{tabular}

Note: Values in bold indicate unacceptable bias estimate 
Table 2

The Comparative Bias Index (CBI) - Model 2

\begin{tabular}{|c|c|c|c|c|c|c|}
\hline \multirow[t]{2}{*}{ Sample size } & \multirow[t]{2}{*}{ Items } & \multicolumn{3}{|c|}{$\begin{array}{c}\text { Comparative Bias Index } \\
\text { CB-SEM }\end{array}$} & \multicolumn{2}{|c|}{$\begin{array}{c}\text { Comparative Bias Index } \\
\text { PLS-SEM }\end{array}$} \\
\hline & & ML & GLS & SLS & PLS & PLSc \\
\hline \multirow[t]{16}{*}{50} & A1 & .950 & .963 & .900 & .964 & .893 \\
\hline & $\mathrm{A} 2$ & 1.000 & .975 & .925 & .918 & .890 \\
\hline & A3 & .950 & .813 & .925 & .913 & .880 \\
\hline & A4 & 1.000 & .988 & .913 & .928 & .913 \\
\hline & B1 & .988 & .925 & .988 & .935 & .973 \\
\hline & B2 & .913 & .750 & .913 & .995 & .904 \\
\hline & B3 & .938 & .850 & .900 & .904 & .880 \\
\hline & B4 & .963 & .763 & .938 & .945 & .930 \\
\hline & $\mathrm{C} 1$ & .938 & .838 & .900 & .909 & .875 \\
\hline & $\mathrm{C} 2$ & .863 & .938 & .913 & .990 & .936 \\
\hline & $\mathrm{C} 3$ & .975 & .975 & .913 & .955 & .865 \\
\hline & $\mathrm{C} 4$ & .963 & .988 & .988 & .900 & .998 \\
\hline & Y1 & .763 & .788 & .750 & .874 & .744 \\
\hline & Y2 & .988 & .800 & .975 & .931 & .958 \\
\hline & Y3 & .950 & .988 & .975 & .888 & .928 \\
\hline & Y4 & .938 & .750 & .963 & .983 & .913 \\
\hline \multirow[t]{16}{*}{100} & A1 & .925 & .938 & .925 & .978 & .924 \\
\hline & A2 & .975 & 1.000 & .950 & .943 & .923 \\
\hline & A3 & .963 & .963 & .963 & .963 & .971 \\
\hline & A4 & 1.000 & .988 & .975 & .945 & .965 \\
\hline & B1 & 1.000 & .975 & .988 & .931 & .966 \\
\hline & B2 & .938 & .950 & .950 & .980 & .953 \\
\hline & B3 & .988 & .988 & .975 & .935 & .960 \\
\hline & B4 & .875 & .863 & .863 & .993 & .861 \\
\hline & $\mathrm{C} 1$ & .988 & .938 & .913 & .935 & .873 \\
\hline & $\mathrm{C} 2$ & .975 & .988 & .963 & .953 & .956 \\
\hline & C3 & .975 & .963 & .963 & .915 & .934 \\
\hline & $\mathrm{C} 4$ & .950 & .913 & .975 & .894 & .984 \\
\hline & Y1 & .863 & .975 & .888 & .971 & .889 \\
\hline & Y2 & .975 & .963 & .988 & .913 & .990 \\
\hline & Y3 & 1.000 & .988 & .975 & .928 & .959 \\
\hline & Y4 & .975 & .950 & .988 & .964 & .999 \\
\hline \multirow[t]{16}{*}{200} & A1 & .975 & .988 & .988 & .941 & .983 \\
\hline & A2 & .963 & .988 & .938 & .955 & .921 \\
\hline & A3 & .975 & 1.000 & .963 & .954 & .955 \\
\hline & A4 & .988 & 1.000 & .988 & .938 & .981 \\
\hline & B1 & .975 & .975 & 1.000 & .916 & .993 \\
\hline & B2 & .975 & .988 & .988 & .956 & .999 \\
\hline & B3 & .950 & .950 & .950 & .970 & .960 \\
\hline & B4 & .938 & .925 & .925 & .971 & .926 \\
\hline & $\mathrm{C} 1$ & .988 & .988 & 1.000 & .940 & .989 \\
\hline & $\mathrm{C} 2$ & .963 & .988 & .963 & .963 & .975 \\
\hline & $\mathrm{C} 3$ & .975 & .988 & .950 & .946 & .939 \\
\hline & $\mathrm{C} 4$ & 1.000 & .988 & 1.000 & .934 & .998 \\
\hline & Y1 & .938 & .988 & .925 & .970 & .914 \\
\hline & Y2 & .988 & .975 & .975 & .925 & .970 \\
\hline & Y3 & .963 & .975 & .950 & .951 & .948 \\
\hline & Y4 & .963 & .963 & .975 & .964 & .990 \\
\hline \multirow[t]{16}{*}{500} & A1 & .938 & .950 & .925 & .965 & .921 \\
\hline & A2 & .950 & .950 & .938 & .973 & .940 \\
\hline & A3 & .950 & .950 & .950 & .966 & .958 \\
\hline & A4 & .975 & .975 & 1.000 & .949 & .998 \\
\hline & B1 & .950 & .938 & .925 & .966 & .913 \\
\hline & B2 & .938 & .938 & .950 & .984 & .955 \\
\hline & B3 & .938 & .938 & .938 & .973 & .929 \\
\hline & B4 & .963 & .975 & .963 & .965 & .970 \\
\hline & $\mathrm{C} 1$ & .950 & .963 & .950 & .965 & .954 \\
\hline & $\mathrm{C} 2$ & .888 & .900 & .900 & .994 & .905 \\
\hline & $\mathrm{C} 3$ & .975 & .975 & .950 & .953 & .931 \\
\hline & $\mathrm{C} 4$ & .925 & .938 & .938 & .985 & .948 \\
\hline & Y1 & .963 & .988 & .975 & .956 & .979 \\
\hline & Y2 & .963 & .975 & .950 & .956 & .936 \\
\hline & Y3 & .963 & .963 & .925 & .960 & .908 \\
\hline & Y4 & .950 & .975 & 1.000 & .964 & .983 \\
\hline
\end{tabular}

Note: Values in bold indicate unacceptable bias estimate 
Table 3

The Comparative Bias Index (CBI) - Model 3

\begin{tabular}{|c|c|c|c|c|c|c|}
\hline \multirow[t]{2}{*}{ Sample size } & \multirow[t]{2}{*}{ Items } & \multicolumn{3}{|c|}{$\begin{array}{c}\text { Comparative Bias Index } \\
\text { PLS-SEM }\end{array}$} & \multicolumn{2}{|c|}{$\begin{array}{c}\text { Comparative Bias Index } \\
\text { PLS-SEM }\end{array}$} \\
\hline & & ML & GLS & SLS & PLS & PLSc \\
\hline \multirow[t]{16}{*}{50} & A1 & .956 & .989 & .967 & .921 & .793 \\
\hline & $\mathrm{A} 2$ & .922 & .911 & .922 & .962 & .791 \\
\hline & A3 & .956 & .956 & .944 & .967 & .996 \\
\hline & A4 & .978 & .956 & .978 & .953 & .967 \\
\hline & B1 & .844 & .922 & .789 & .947 & .864 \\
\hline & B2 & .867 & .844 & .856 & .893 & .803 \\
\hline & B3 & .878 & .900 & .956 & .974 & .996 \\
\hline & B4 & .967 & .989 & .967 & .938 & .827 \\
\hline & $\mathrm{C} 1$ & .911 & .878 & .889 & .970 & 1.000 \\
\hline & $\mathrm{C} 2$ & .911 & .922 & .889 & .880 & .832 \\
\hline & $\mathrm{C} 3$ & .967 & .811 & .978 & .929 & .769 \\
\hline & $\mathrm{C} 4$ & .933 & .811 & .911 & .978 & .891 \\
\hline & Y1 & .878 & .944 & .833 & .777 & .661 \\
\hline & Y2 & .956 & .811 & .956 & .950 & .851 \\
\hline & Y3 & .933 & .900 & .856 & .989 & .824 \\
\hline & Y4 & .822 & .778 & .933 & .904 & .967 \\
\hline \multirow[t]{16}{*}{100} & A1 & .911 & .933 & .956 & .979 & .972 \\
\hline & $\mathrm{A} 2$ & .867 & .889 & .833 & .931 & .809 \\
\hline & A3 & .978 & .978 & .978 & .996 & .984 \\
\hline & A4 & .956 & .978 & .944 & .994 & .947 \\
\hline & B1 & .856 & .878 & .833 & .933 & .812 \\
\hline & B2 & .789 & .789 & .778 & .859 & .796 \\
\hline & B3 & .878 & .878 & .900 & .958 & .908 \\
\hline & B4 & .989 & 1.000 & 1.000 & .993 & .994 \\
\hline & $\mathrm{C} 1$ & .922 & .967 & .922 & .977 & .917 \\
\hline & $\mathrm{C} 2$ & .889 & .967 & .867 & .931 & .857 \\
\hline & $\mathrm{C} 3$ & .900 & .911 & .911 & .957 & .911 \\
\hline & $\mathrm{C} 4$ & .922 & .944 & .933 & .979 & .941 \\
\hline & Y1 & .911 & .911 & .933 & .971 & .941 \\
\hline & Y2 & .989 & .978 & .967 & .988 & .961 \\
\hline & Y3 & .967 & 1.000 & .944 & .999 & .919 \\
\hline & Y4 & .944 & .944 & .967 & .988 & .980 \\
\hline \multirow[t]{16}{*}{200} & A1 & .978 & .989 & .989 & .983 & .974 \\
\hline & A2 & .956 & .967 & .933 & .994 & .931 \\
\hline & A3 & .978 & .989 & .978 & .989 & .986 \\
\hline & A4 & .989 & 1.000 & .967 & .982 & .951 \\
\hline & B1 & .911 & .922 & .889 & .962 & .892 \\
\hline & B2 & .900 & .911 & .867 & .952 & .856 \\
\hline & B3 & .900 & .922 & .933 & .966 & .952 \\
\hline & B4 & .967 & .967 & .978 & 1.000 & .972 \\
\hline & $\mathrm{C} 1$ & .967 & .978 & .956 & 1.000 & .951 \\
\hline & $\mathrm{C} 2$ & .911 & .956 & .900 & .954 & .889 \\
\hline & C3 & .911 & .922 & .922 & .971 & .927 \\
\hline & C4 & .944 & .967 & .956 & .989 & .958 \\
\hline & Y1 & .922 & .933 & .944 & .979 & .956 \\
\hline & Y2 & .978 & .967 & .978 & .989 & .972 \\
\hline & Y3 & .967 & .967 & .944 & .998 & .927 \\
\hline & Y4 & .956 & .956 & .967 & .996 & .969 \\
\hline \multirow[t]{16}{*}{500} & A1 & .967 & .978 & .978 & .992 & .983 \\
\hline & A2 & .967 & .978 & .944 & .993 & .941 \\
\hline & A3 & .967 & .967 & .978 & .993 & .988 \\
\hline & A4 & 1.000 & 1.000 & .989 & .980 & .983 \\
\hline & B1 & .956 & .967 & .967 & .998 & .974 \\
\hline & B2 & .956 & .956 & .944 & .993 & .939 \\
\hline & B3 & .967 & .967 & .956 & .998 & .953 \\
\hline & B4 & .978 & .978 & .978 & .989 & .980 \\
\hline & $\mathrm{C} 1$ & .967 & .978 & .956 & .992 & .952 \\
\hline & $\mathrm{C} 2$ & .922 & .944 & .911 & .972 & .913 \\
\hline & $\mathrm{C} 3$ & .933 & .933 & .944 & .986 & .942 \\
\hline & $\mathrm{C} 4$ & .956 & .956 & .967 & .991 & .973 \\
\hline & Y1 & .956 & .956 & .967 & .999 & .973 \\
\hline & Y2 & .978 & .978 & .978 & .991 & .978 \\
\hline & Y3 & .978 & .989 & .956 & .988 & .949 \\
\hline & Y4 & .956 & .956 & .956 & .997 & .964 \\
\hline
\end{tabular}

Note: Values in bold indicate unacceptable bias estimate 
The loading for Model 1 was set as 0.7 for every item underlying the respective constructs. Table 1 shows the results for Model 1. Among the CB-SEM estimators, SFLS consists of 5 and ML consists of 4 low CBI values $(<0.8)$ indicators for a small sample size $(n=50)$. GLS consisted of 9 low CBI values $(<0.8)$ indicators. PLS generates better CBI values in most of the indicators, compared to PLSc and CB-SEM estimators when the sample size is small. This result implies that at a low sample size $(n=50)$, CB-SEM estimators (ML, GLS, SFLS) generated several biased indicators loading estimates similar to PLSc because the latter mimics a CB-SEM estimator (Dijkstra \& Henseler, 2015). Contrarily, CB-SEM estimators comprised of only 1 to 3 low CBI values $(<0.8)$ indicator when the sample size increased $(n=100)$. This finding attests that CB-SEM estimators require at least 100 sample sizes to produce good estimates. This finding was also applicable to PLSc. At a large sample size $(n \geq 200)$, ML and GLS do not consist of any undesirable bias estimates $(<0.8)$. Surprisingly, there seem to be no undesirable bias estimates $(<0.8)$ in PLS. A thorough observation of CBI values show that GLS produces a better estimate compared to ML and PLS in most indicators, with CBI values closer to 1.0. As the sample size increases (i.e., $n=500$ ), none of the estimators in CB-SEM and PLS-SEM generate any undesirable bias estimates $(<0.8)$. A closer look at their CBI values reveals that for large sample sizes, the CB-SEM estimators produce a high value compared to PLS-SEM. This finding proves that PLSc requires a large sample $(n \geq 100)$ to generate a better estimate. The biasness of indicator loading estimates in ML, GLS, SFLS, and PLSc at a low sample size might be due to their underestimation compared to PLS. The indicator loadings estimated by the CBI for Model 2 is shown in Table 2. Every item loading underlying the respective constructs for Model 2 was set as 0.8. Based on the results, CB-SEM estimators consist of merely 1 low CBI value $(<0.8)$ except GLS at a low sample size $(\mathrm{n}=50)$, similar to PLSc. Interestingly, PLS does not produce any undesirable bias estimates $(<0.8)$ across all sample sizes $(n=50,100,200,500)$. In a large sample $(n \geq 200)$, the total number of low bias indicator estimates $(C B I>0.9)$ in GLS and SFLS is higher than ML, indicating that the use of other estimation methods can be considered despite ML being appropriate for large sample sizes. Similarly, PLS and PLSc estimate indicator loadings with high CBI values $(>0.9)$ across large sample sizes $(n=200,500)$. For Model 3, every item loading underlying the respective constructs was set as 0.9 . The result is shown in Table 3. Among CB-SEM estimators, it is reported that ML does not produce any undesirable bias estimates $(<0.8)$ for a low sample size. Having said that, at low sample size, ML shows a better CBI performance than PLS for Model 3. Meanwhile, PLSc consists of 4 low CBI values $(<0.8)$ indicators, indicating that among other estimators, PLSc generates several unacceptable bias estimates $(<0.8)$. For 100 sample sizes, we observed a contradictory finding where ML generates 1 unacceptable bias estimate $(<0.8)$ and conversely for PLS. At the same time, other estimators in CB-SEM and PLS-SEM consist of 1 low CBI values $(<0.8)$ indicator. In a large sample $(n \geq 200)$, ML and PLS produce low bias estimates $(>0.9)$ in all indicators.

\section{Discussion}

The current study examined the performance of several estimation methods in terms of CBI values. We used simulation to create data with various sample sizes employing a simple model following specific criteria (e.g., normal, complete data). Based on the findings, we derive some conclusions. As stated, the true loadings of indicators for the three models are homogenous between 0.7 to 0.9 . At sample size 50 , where the items' true indicator loading was set as 0.7 , PLS consists of only 1 low CBI value and therefore performs markedly better than PLSc and CB-SEM estimators. However, at sample size 100, ML and GLS generate 1 unbiased indicator loading estimate while PLS shows consistent results. At sample size $n \geq 200$, ML, GLS, and PLS do not consist of any low CBI values. Therefore, we infer that when data of true loading 0.7 is to be simulated, the study can be conducted by using GLS as an alternative fitting function in CB-SEM and PLS estimator.

For Model 2, PLS is capable of providing accurate estimates for all the sample sizes. This finding suggests that PLS produces unbiased estimation across all sample sizes when the actual indicator loading is high (i.e. 0.8). In contrast, PLSc and CB-SEM estimators proved to perform notably better when the indicator loadings are high (i.e. 0.8$)$ across large sample sizes $(n=100$, 200, 500). However, among all the estimators for Model 3, ML does not comprise low CBI values $(<0.8)$ and PLSc produces several biased loading estimates at a low sample size. Meanwhile, GLS, SFLS, and PLS were observed to generate 1 biased loading estimate, suggesting that when a population indicator loading is high (e.g., 0.9), ML estimation's performance is superior compared to others. Contrarily, at 100 samples, ML shows inconsistent results; it consists of 1 low CBI value $(<0.8)$. However, the CBI for each loading indicator using PLS is high, with no low CBI values found over large sample sizes $(n \geq$ $100)$.

From the findings, we can conclude that when true indicator loadings are high (i.e. 0.9), the indicator loadings are underestimated and therefore fall within the range of acceptable bias. At large sample sizes $(n \geq 200)$, we infer that when it refers to simulation study with the population indicator loadings greater than 0.8 , one can consider using CB-SEM and PLS-SEM estimators to generate better parameter estimates. An indicator loading value of 0.8 indicates great internal consistency (Rahlin et al., 2019). If the true indicator loadings are 0.7 , ML, GLS, and PLS are better alternatives for a large sample size ( $n \geq 100)$. Consistent with Aimran et al. (2017a), when the actual indicator loadings are consistently high (e.g., $\geq 0.8$ ), PLS can be considered as a good estimator. The biases of indicator loading estimates in ML, GLS, SFLS and PLSc at low sample size might be due to its underestimation compared to PLS. The findings of this study also prove that for the cases of 50 samples and the true indicator loading being extremely high (e.g., 0.9), PLSc clearly underestimates the true value of indicator loading, thus producing unacceptable bias estimates. This issue may have arisen due to the consequences of measurement error propagation on parameter estimates (Afthanorhan et al., 2021). When estimating the indicator loadings for confirmatory purposes, 
one should consider using CB-SEM rather than PLSc because the former produces more accurate estimates for indicator loadings (Afthanorhan et al., 2020).

This study is not without limitations. The comparison of this model's implementations to CB-SEM and PLS-SEM is not certainly expected to be generalized to all models because the conclusions are derived from the model within the scale of this study. However, such will raise scholars' awareness of several critical issues that may emerge and should be pondered when choosing the relevant SEM technique for their research.

\section{Conclusion}

From this study, we conclude that PLS is a good estimator if the actual indicator loadings are consistently high (e.g., $\geq 0.8$ ). The biases of indicator loading estimates in ML, GLS, SFLS, and PLSc at low sample size might be due to its underestimation compared to PLS. On the other hand, the indicator loadings are underestimated hence fall within the permissible bias range when true indicator loadings are high (i.e., 0.9).

\section{Acknowledgements}

The authors would like to express their gratitude to the Research Management Centre, Universiti Teknologi MARA, Shah Alam for the funding of the publication of this paper.

\section{References}

Afthanorhan, A., Awang, Z., \& Aimran, N. (2020). An extensive comparison of cb-sem and pls-sem for reliability and validity. International Journal of Data and Network Science, 4(4), 357-364.

Afthanorhan, A., Awang, Z., Aimran, N., \& Arifin, J. (2021). An Extensive Comparison Between CBSEM and Consistent PLS-SEM On Producing the Estimates of Construct Correlation in Applied Research. Journal of Physics: Conference Series, 1874(1), 012083.

Aimran, A. N., Ahmad, S., Afthanorhan, A., \& Awang, Z. (2017a). The assessment of the performance of covariance-based structural equation modeling and partial least square path modeling. AIP Conference Proceedings, 1842.

Aimran, A. N., Ahmad, S., Afthanorhan, A., \& Awang, Z. (2017b). The development of comparative bias index. AIP Conference Proceedings, 1870.

Ainur, A. K., Sayang, M. D., Jannoo, Z., \& Yap, B. W. (2017). Sample size and non-normality effects on goodness of fit measures in structural equation models. Pertanika Journal of Science and Technology, 25(2), 575-586.

Andreassen, T. W., Lorentzen, B. G., \& Olsson, U. H. (2006). The impact of non-normality and estimation methods in SEM on satisfaction research in marketing. Quality and Quantity, 40(1), 39-58.

Arbuckle, J. L. (2011). IBM SPSS Amos 20 user's guide. Amos Development Corporation, SPSS Inc.

Awang, Z. (2015). SEM made simple: A gentle approach to learning Structural Equation Modeling. MPWS Rich Publication.

Dijkstra, T. K. (2010). Latent Variables and Indices: Herman Wold's Basic Design and Partial Least Squares. in Handbook of Partial Least Squares: Concepts, Methods, and Applications,V. E. Vinzi, W. W. Chin, J. Henseler, and H. Wang (eds.), New York: Springer, pp. 23-46.

Dijkstra, T. K., \& Henseler, J. (2015). Consistent partial least squares path modeling. MIS Quarterly: Management Information Systems, 39(2), 297-316.

Hair., Matthews, L. M., Matthews, R. L., \& Sarstedt, M. (2017). PLS-SEM or CB-SEM: updated guidelines on which method to use. International Journal of Multivariate Data Analysis, 1(2), 107.

Hair, Risher, J. J., Sarstedt, M., \& Ringle, C. M. (2018). The Results of PLS-SEM Article information. European Business Review, 31(1), 2-24.

Henseler, J., Ringle, C. M., \& Sinkovics, R. R. (2009). The use of partial least squares path modeling in international marketing. In R. R. Sinkovics \& P. N. Ghauri (Eds.), Advances in international marketing (Vol. 20, pp. 277-320). Bingley: Emerald.

Henseler, J., Hubona, G., \& Ray, P. A. (2016). Using PLS path modeling in new technology research: Updated guidelines. Industrial Management and Data Systems, 116(1), 2-20.

Maydeu-Olivares, A. (2017). Maximum likelihood estimation of structural equation models for continuous data: Standard errors and goodness of fit. Structural Equation Modeling: A Multidisciplinary Journal, 24(3), 383-394.

McDonald, R. P. (1996). Path analysis with composite variables. Multivariate Behavioral Research, 31(2), $239-270$.

Newsom, J.T. (2018). Alternative Estimation Methods Remember. Psy 523/623 Structural Equation Modeling, 1-3.

Rahlin, N. A., Awang, Z., Afthanorhan, A., \& Aimran, N. (2019). Antecedents and consequences of employee safety climate in the small manufacturing enterprises: Translation, validation and application of the generic safety climate questionnaire. International Journal of Innovation, Creativity and Change, 7(10), 307-328.

Sarstedt, M., Ringle, C.M., Henseler, J., \& Hair, J.F. (2014). On the emancipation of PLS-SEM: a useful tool for family 
business researchers. Journal of Family Business Strategy, 5(1), 105-115.

Schamberger, T., Schuberth, F., Henseler, J., \& Dijkstra, T. K. (2020). Robust partial least squares path modeling. Behaviormetrika, 47(1), 307-334.

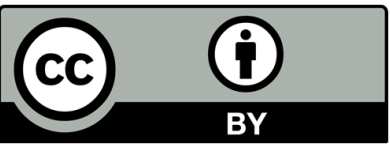

(C) 2022 by the authors; licensee Growing Science, Canada. This is an open access article distributed under the terms and conditions of the Creative Commons Attribution (CC-BY). license (http://creativecommons.org/licenses/by/4.0/). 